\title{
The role of liver in metabolism: an updated review with physiological emphasis
}

\author{
Zaenah Zuhair Alamri*
}

Department of Biology, Faculty of Science, Jeddah University, Saudi Arabia

Received: 15 August 2018

Revised: 15 September 2018

Accepted: 19 September 2018

\section{*Correspondence to:}

Dr. Zaenah Zuhair Alamri, Email: dr.zaenahza@gmail.com

Copyright: (C) the author(s), publisher and licensee Medip Academy. This is an openaccess article distributed under the terms of the Creative Commons Attribution NonCommercial License, which permits unrestricted noncommercial use, distribution, and reproduction in any medium, provided the original work is properly cited.

\begin{abstract}
Liver plays an essential role in metabolism and has an important role in preserving and regulating the levels of lipid, glucose in the body as well as energy metabolism. Among the important functions performed by the liver is maintaining of blood glucose levels under different conditions through group of processes included; glycolysis, glycogenesis, glycogenolysis, gluconeogenesis. The absorbed free fatty acids and those derived from the adipose tissue reach the liver and are utilized for energy, membrane synthesis, or stored as triglyceride. In addition, the liver has a crucial role in keeping homeostasis of body level of cholesterol. Regarding protein metabolism, urea cycle occurs in the liver through the action of urea cycle enzymes to produce urea in order to get rid of the toxic ammonia. In the liver, cholesterol is utilized for bile acids synthesis through a complicated process. These bile acids are considered essential in order to absorb and transport of lipid-soluble vitamins dietary and fat in the diet as well as clearance of drugs, toxic substances and xenobiotics. Adding to these hepatic functions is hepatic detoxification where liver metabolizes a various type of drugs to make soluble execretable compounds. In conclusion, the liver has so important metabolic functions which if impaired will resulted in many liver diseases and might progress to more dangerous conditions such as liver fibrosis or cirrhosis.
\end{abstract}

Keywords: Bile acids, Circadian, Carbohydrates, Drugs, Liver, Lipids, Metabolism, Proteins, Physiology

\section{INTRODUCTION}

The liver is considered the second-largest organ in the body and its weight ranged from 1.5 to $2.5 \%$ of the lean body weight. It performs vascular, immunological, metabolic, and secretory and excretory functions. ${ }^{1}$ About $30 \%$ of the cardiac output is directed to the liver which exceptionally receives both arterial blood from the hepatic artery and venous blood from the portal vein. The latter supplies about 70 to $75 \%$ of hepatic blood flow but only $50 \%$ of oxygen supply and it takes the blood directly from the gut to the liver. This allows for first pass metabolism and makes the liver prone to the ingested drugs as they are absorbed from the intestine. ${ }^{2}$
It plays an essential role in metabolism and has an important role in preserving and regulating the levels of lipid, glucose in the body as well as energy metabolism. Among the important functions performed by the liver is maintaining of blood glucose levels during fasting by releasing glucose from glycogen and synthesis of glucose from amino acids.

Fat and cholesterol absorbed from the diet were brought to and accumulated in the liver which produces fatty acids and cholesterol from acetyl-CoA come from glucose. In the liver, excess fatty acids could be converted to ketone bodies that supply energy to brain and muscle in case of starvation. ${ }^{3}$ 


\section{REVIEW OF LITERATURE}

\section{Circadian control of liver metabolic functions}

The liver is a key metabolic center as it masters many essential metabolic functions for example nutrient metabolism, synthesis of essential serum components, detoxification. All these vital hepatic functions should be adjusted to "a rhythmically changing systemic environment". The liver, similar to other body organs, has a circadian clock which represents an internal timing system works to adjust the physiological functions to their appropriate time of day. The liver utilizes this system to antedate recurrent general and ecological alterations and function. ${ }^{4}$

The circadian clock is defined as "an endogenous timekeeper that permits organisms to match their biological time with the cyclic environment provided by $24 \mathrm{~h}$ solar days. Hence, circadian rhythms in physiology and behavior are evolutionary conserved processes present in almost all living forms". 5 A well-timed planned physiology conducted by the circadian clock provides organisms the capacity to anticipate and prepare for recurrent environmental variations occur during the day and the night, and it also coordinates temporal partition of incompatible metabolic processes. ${ }^{6}$

\section{Carbohydrate metabolism}

When the meal is digested, and glucose is absorbed from the intestine into the circulation, blood glucose levels in portal vein is elevated to more than $10 \mathrm{mM}$, glucose moves into the hepatocytes via liver glucose transporter 2 (GLUT2). The liver transforms the excess glucose into glycogen. This process is named "glycogenesis" which is stimulated by insulin which activates and dephosphorylates glycogen synthase. ${ }^{1}$ Two to three hours after the meal (the postprandial period), blood glucose concentrations drops to the normal level and glycogenolysis is initiated by decreased insulin/glucagon ratio whereas glucagon hormone increases adenylyl cyclase action to upgrade cyclic Adenosine monophosphate levels that enhance protein kinase $\mathrm{A}^{3}$ (Figure 1).

Insulin does not spur GLUT2 in the liver, instead it catalyzes the GLUT4 exists in the muscular and fatty tissues by activating translocation of intracellular GLUT4 to the cell surface. Liver cell stowed fatty acids, glucose and ketone bodies and conveyed to the other tissues in order to generate energy. ${ }^{3}$

Glycolysis, oxidizing glucose to generate ATP for energy metabolism only utilizes approximately $20-30 \%$ of glucose internalized by the liver in order to produce. The residual glucose is used for glycogen, fatty acids, and ketone bodies composition. Insulin activates liver pyruvate Kinase by dephosphorylation. During feeding, insulin spurs glycolysis by dephosphorylation of phosphofructokinase 2 (PFK2) with subsequent phosphorylation of fructose-6-phosphate (F-6-P) into F-2, 6-P, a chief controller of glycolysis in the liver. In fasting, PFK2 is inactivated by protein kinase A (PKA). Liver protein kinase is stimulated by fructose 1,6-bis $\mathrm{P}$ and transforms phosphoenolpyruvate (PEP) into pyruvate. ${ }^{3}$

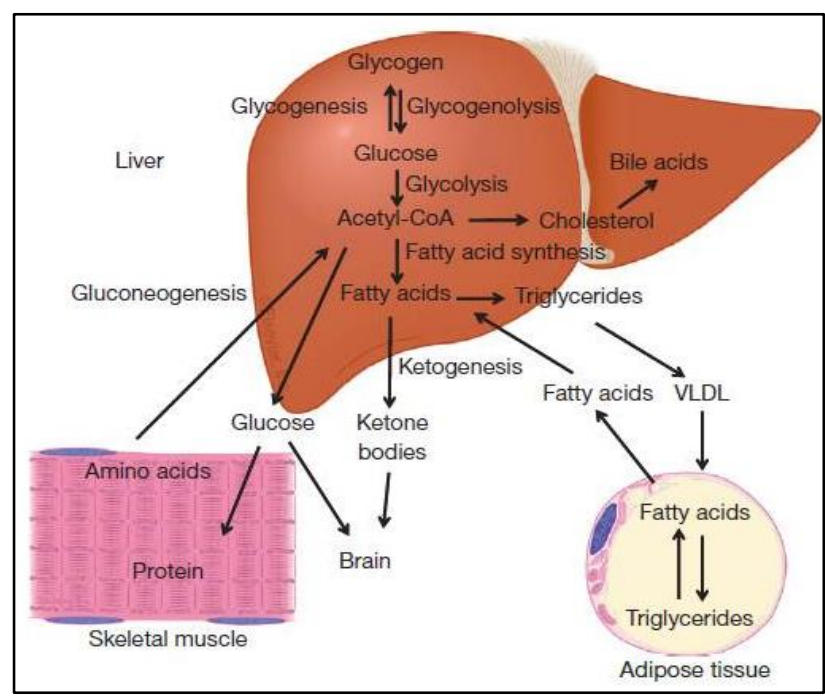

Figure 1: Major metabolic functions of the liver (Chiang et al). ${ }^{3}$

During the 'normal' fasting state, when glucose levels decreased, liver starts the breakdown of glycogen into glucose molecules that can be transported to other tissues for generating energy in the form of adenosine triphosphate (ATP). This process is named "glycogenolysis". ${ }^{1}$

In certain conditions as during starvation, the liver glycogen reserve is depleted then the liver synthesizes glucose from amino acids (essentially muscular alanine), lactate, and glycerol to build up glycogen reserves in the body. This process is known as "gluconeogenesis". ${ }^{1}$ Gluconeogenesis is activated during fasting by glucagon and inhibited during feeding by insulin. Phosphoenolpyruvate carboxy kinase is considered a crucial regulating enzyme in the transformation of oxaloacetate to phosphoenolpyruvate then into G-6-P in an inverted order of glycolysis reactions. In the next step, G6Pase transforms G-6-P into glucose. ${ }^{3}$

\section{Circadian control of carbohydrate metabolism}

Glucose homeostasis is being regulated mainly by the liver besides other organs including the brain, pancreas, and skeletal muscle. ${ }^{7}$ This regulation is performed through certain procedures like insulin and glucagon secretion as well as glucose synthesis and uptake that were documented to show circadian rhythm clearly distinct from nutrient signaling. ${ }^{8,9}$ It was described that "rhythmic insulin secretion is controlled by pancreatic clocks in rodents and humans, which seem to be vital for pancreatic function". ${ }^{10,11}$ The circadian clocks in the suprachiasmatic 
nucleus and the liver utilize altered mechanisms to produce antiphasic rhythms of glucose metabolism, which together induces nearly reliable levels of blood glucose during the 24 hours. $^{7}$

\section{Protein metabolism}

Free amino acids, the end product of dietary protein digestion and those resulted from the cellular protein breakdown might go through further degradation in the cell. This occurs through removal of the alpha-amino group which transformed into ammonia and combined into urea to be excreted. The urea cycle occurs in the liver through the action of urea cycle enzymes. In this cycle two molecules of ammonia and one molecule of carbon dioxide are utilized to produce urea in order to get rid of the toxic ammonia. The significance of the disposal of ammonia into urea can be perceived in patients suffering from ornithine transcarbamylase deficiency, one of urea cycle disorders, this which are liable to accumulate ammonia. Most of urea (about $80 \%$ ) produced in the liver is excreted in the urine through kidneys although not all the amount generated in the liver excreted in the urine. This is because the other part is transformed by colonic bacteria into ammonia, which may be important in de novo protein synthesis in the body, mostly during starvation. The other part of urea (about 10\%) is excreted in the faeces. ${ }^{12}$

Urea cycle enzymes in liver are regulated by glucagon, insulin, and glucocorticoids while, in other cells are regulated by a wide range of pro- and anti-inflammatory cytokines and other agents. ${ }^{13}$ Impaired activity of one or more of these enzymes or transporters by mutations results in inborn errors of nitrogen metabolism and detoxification pathway. ${ }^{14}$

Morlion et al, described protein metabolism during the times of substantial physical stress to be shifted more towards amino acid oxidation with a net flux from muscle to liver. In such conditions, amino acids are used for gluconeogenesis as well as providing for production of acute phase proteins in the liver where glucogenic amino acids transform into "glucose precursors pyruvate, aketoglutarate, fumarate, oxaloacetate or succinyl-CoA". ${ }^{15}$

\section{Lipid metabolism}

After the digestion and absorption of the dietary lipids, the absorbed free fatty acids as well as those derived from the adipose tissue reach the liver and are utilized for energy, membrane synthesis, or stored as triglyceride (TG).

Free fatty acids (FFAs) diffuse to inside the hepatocytes. Adding to that the fatty acid transport protein (FATP) and fatty acid translocase (FAT) that facilitate the entrance of long-chain fatty acids. ${ }^{16}$

"Inside the hepatocytes, FFA binds to fatty acid binding protein (FABP)-1 which directs FFAs from cytoplasm to different compartments within the cell either for metabolism or for regulation of gene expression through interaction with transcription factors, such as peroxisome proliferator-activated receptor (PPAR) $\alpha$, thereby protecting liver from lipotoxicity (Figure 2). ${ }^{17}$

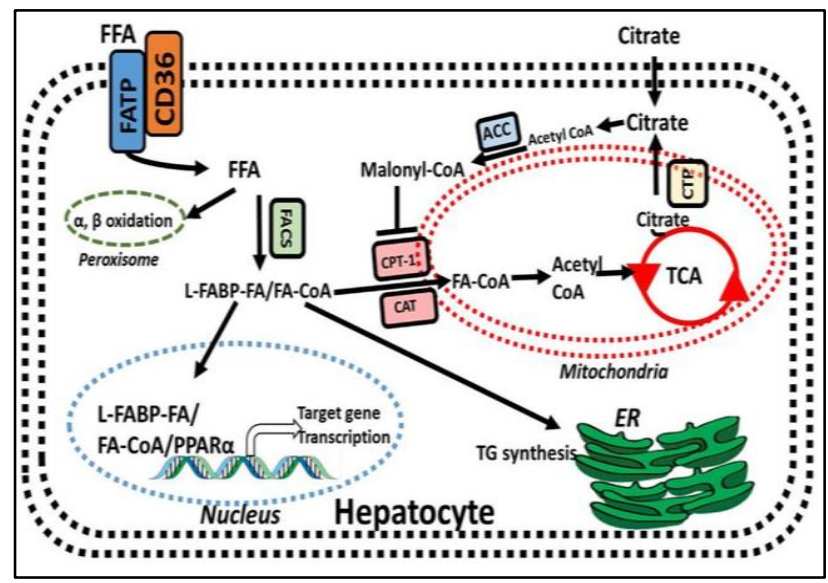

Figure 2: Fate of free fatty acids (FFA) in hepatocytes. (Chiang et al). ${ }^{3}$

Shi et al, after conducted their study on Chinese adults concluded that "serum FABP1correlated with obesity, insulin resistance, hypertriglyceridemia and low HDL cholesterol". These findings proposed that FABP1 has a crucial role in lipid metabolism in liver as well as in hepatic cell protection thus it becomes a target for drug discovery to prevent oxidative stress- and lipotoxicityinduced hepatic damage. ${ }^{18}$ Lipid metabolism in liver was reported to be affected also by adipocytes-FABP (AFABP) which affects insulin sensitivity, as well as the circulating non-esterified fatty acid levels. It was stated that "mice lacking A-FABP in adipocytes showed reduced lipolysis and were more insulin sensitive compared to obese control mice. ${ }^{19}$

De novo lipogenesis, synthesis of faty acides from nonlipid precursors such as glucose, is a multienzymatic process that takes place in the liver when both glucose and insulin levels are elevated. In this process, "excess glucose is oxidized to pyruvate and converted to citrate via the Krebs cycle in the mitochondria. Citrate can be either metabolized in the mitochondria through the Krebs cycle to produce ATP or transported to the cytosol and used to synthesize fatty acids. The transportation of citrate to the cytosol is mediated by its transporter, citrate transporting protein (CTP), through electroneutral exchange". ${ }^{20}$ In the healthy conditions, de novo lipogenesis contributes to only from 5 to $10 \%$ of the TG in the liver; while this percent is elevated to exceed $25 \%$ in NAFLD patients signifying that this process may be a main contributing factor in the occurrence of NAFLD in humans. ${ }^{21,22}$

Normally, FFA undergo oxidation in the liver to generate energy through $\alpha, \beta$ and $\omega$ oxidation. The latter takes place in microsomes whereas $\alpha, \beta$ oxidation takes place in peroxisomes and mitochondria. Mitochondrial $B$ oxidation is considered the main FFA disposal pathway in liver. ${ }^{23}$ 
Fatty acid oxidation in the liver is initiate by fatty acylCoA synthase (FACS)-induced activation as well as transportation of FA-CoA into the mitochondria by FABP. It was stated that L-FABP is necessary for high rates of hepatic fatty acid oxidation under fasting conditions. ${ }^{24}$

Secretion of TG from the liver after being assembled with Apoprotein B occurs in the liver in the form of very-low density lipoproteins (VLDL). The regulating enzyme in this process is the microsomal triglyceride transfer protein (MTTP). Insulin directly regulates MTTP expression. Not only that, insulin also limits apoB synthesis and accelerates its degradation, which all gives rise to decreased VLDL secretion and hence TG export from the liver. In cases of insulin deficiency or resistance, expression of MTTP is up-regulated, and consequently VLDL secretion increased. ${ }^{25}$

\section{Circadian control of lipid metabolism}

It was reported that lipids are potential regulators of circadian rhythmicity. On the other hands, the circadian clock controls most aspects of lipid metabolism in the liver. ${ }^{26}$ It was stated that "circadian lipid metabolism is not surprisingly controlled to a large extent by the clockdependent regulation of key enzymes and transcription factors".

Adding to that the enzymes of the glycerol 3-phosphate pathway as well as the enzymes that regulate fatty acid synthesis that showed rhythmic expression. Similarly, factors that regulates lipid such as "peroxisome proliferator-activated receptor (PPAR)" are expressed in rhythmic patterns in liver cells. ${ }^{27}$ Indirect proof of the regulation of circadian clock utilities by lipids mainly originates from studies on "nuclear receptors that are embedded within the core oscillator mechanism". ${ }^{26}$

\section{DISCUSSION}

There are many other metabolic functions of the liver. Among this function are cholesterol metabolism, bile acids metabolism and drug detoxification.

\section{Cholesterol metabolism}

Cholesterol represents a main constituent of the biological membranes. Sex hormones, steroids, and bile acids are all derived from cholesterol. The daily cholesterol input in human is $100 \mathrm{gm}$ which represents approximately $1-1.5 \%$ of the entire cholesterol amount as about $300-500 \mathrm{mg}$ of cholesterol are absorbed daily from diets while $600-900 \mathrm{mg}$ of cholesterol is produced per day.

Liver utilizes cholesterol to synthesize from 500 to $600 \mathrm{mg}$ of bile acids that enable the secretion of about $600 \mathrm{mg}$ of cholesterol into the bile. Cholesterol input is accurately well-adjusted o cholesterol output in order to maintain whole body cholesterol homeostasis as simply a minor quantity of cholesterol is being utilized for cell membrane and steroid hormones formation (Figure 3 ). ${ }^{3}$

"Liver obtains cholesterol through dietary absorption, LDL receptor-mediated uptake and de novo synthesis. Cholesterol is synthesized from acetyl-CoA through a very complicated pathway involving more than 25 enzymes. The rate-limiting step of cholesterol synthesis is catalysed by $\mathrm{HMG}-\mathrm{CoA}$ reductase. ${ }^{3}$

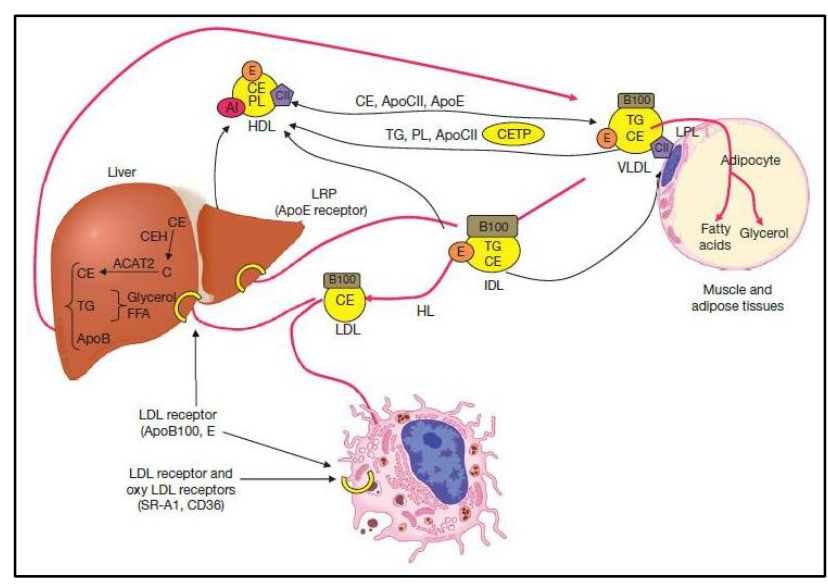

Figure 3: Transport cholesterol and triglycerides from the liver to other tissues (Chiang et al). ${ }^{3}$

\section{Bile acids metabolism}

Bile acids is considered as "physiological detergents that are required for absorption and transport of dietary fats and lipid-soluble vitamins, and disposal of toxic metabolites, drugs, and xenobiotics". Cholic acid and chenodeoxycholic acid are considered the two main primary bile acids formed by the human liver. ${ }^{3}$

Enterohepatic circulation of bile acids is considered the physiological pathway that controls bile acid formation through a feedback order. This circulation is important in order to transport the nutrients absorbed from the intestine to the liver to be metabolized and distributed to other body organs. It is also responsible for the reabsorption of most of the bile acids (95\%) from the ileum and only $5 \%$ bile acids pass in the feces. ${ }^{3}$

\section{Circadian control of bile acids metabolism}

The liver is considered the main organ in which cholesterol is converted into bile acids. The latter facilitates nutrients absorption in the intestine. Bile acid homeostasis is mainly oversaw by a feedback mechanism including FXR, fibroblast growth factor 15 (FGF15), and small heterodimer partner (SHP), but like the homeostatic regulation of blood glucose levels, the circadian clock allows supplementary regulatory mechanisms. ${ }^{28}$ It was stated that "The cycling transcription factor KLF15, which functions as a repressor of the FXR-FGF15 signaling pathway, also controls bile acid synthesis. Particularly, 
diurnal rhythms of bile acid synthesis have also been evident in humans". ${ }^{29}$

\section{Drug detoxification}

The liver metabolizes a various type of drugs, with the aim to make it water soluble excretable compounds in the bile. This processed named hepatic drug detoxification which simply includes two phases. The first phase 1 includes oxidation, reduction and hydrolysis reactions and is mediated by cytochrome P450 while the second phase includes conjugative reactions. The cytochrome $\mathrm{P} 450$ is a family of enzymes located essentially in the liver. They are responsible for the oxidation and reduction reactions occurs during phase 1 by using iron in order to enhance the water solubility of drugs to enhance excretion. A number of non-cytochrome $\mathrm{P} 450$ dependent reactions occur in the liver, for example oxidation of dopamine and alcohol, and hydrolysis of amides and esters. ${ }^{2}$

Most of the anesthetics and intensive care drugs are administered intravenously while others might be given orally or nasogastrically and absorbed enterally. In this case, the absorption will be affected in many conditions like delayed gastric emptying, diarrhoea and liver failure in which gastric transit time is prolonged.

In liver failure the degree of metabolism will be decreased, consequently the extraction ratio will also be decreased, and more drug will reach the systemic circulation, thus increasing bioavailability. In a cirrhotic liver, blood is diverted directly into the systemic circulation by-passing the liver through the portovenous shunting in the varices with subsequent reduction in the first pass metabolism. Splanchnic vasoconstriction and reduced liver blood flow occurs after the use of vasopressors on intensive care is behind the reduction of drug metabolism by the liver. ${ }^{30}$

\section{Circadian control of hepatic drug detoxification}

Among the functions of the liver is the clearance of the toxic substances from the blood. This occurs when these substances were converted to water-soluble metabolites in order to get rid of it. Circadian clock controls the different phases of the hepatic d clearance of the toxic substances. In order to be detoxified, xenobiotics should be bind first to the nuclear receptors then the transcription of the detoxification pathways is activated. rhythmic expression levels of nuclear receptor genes control the circadian regulation in the liver. ${ }^{31}$

The different types of cytochromes as well as the different proteins involved in substrate oxidation occurs during the first phase of hepatic detoxification all are regulated in a circadian mode. At the time of food ingestion, the expression peak of such regulator reaches their maximum. During the second phase of the hepatic detoxification, the expression of the conjugating enzymes that transform toxins into water soluble substances is also rhythmically controlled. Excretion is started via transporter proteins of different types during the third phase of the hepatic detoxification. ${ }^{32}$ Gachon et al, reported that "the master regulators of all classes of detoxification enzymes are the liver-specific PAR bZIP proteins which are rhythmically activated through CLOCK and BMAL1 binding sites in their promoters". ${ }^{33}$

\section{Funding: No funding sources \\ Conflict of interest: None declared \\ Ethical approval: Not required}

\section{REFERENCES}

1. Mitra V, Metcalf J. Metabolic functions of the liver. Anaesthesia Intensive Care Med. 2009;10(7):334-5.

2. Vaja R, Ghuman N. Drugs and the liver. Anaesthesia Intensive Care Med. 2017;19(1):30-4.

3. Chiang J. Liver physiology: Metabolism and detoxification. In: Linda M. McManus, Richard N. Mitchell, editors. Pathobiology of human disease. San Diego: Elsevier. 2014:1770-1782.

4. Reinke H, Asher G. Circadian clock control of liver metabolic functions. Gastroenterol. 2016 Mar 1;150(3):574-80.

5. Peek CB, Ramsey KM, Marcheva B, Bass J. Nutrient sensing and the circadian clock. Trends Endocrinol Metab. 2012 Jul 1;23(7):312-8.

6. Zhang EE, Kay SA. Clocks not winding down: unravelling circadian networks. Nature Rev Mol Cell Biol. 2010 Nov;11(11):764.

7. Lamia KA, Storch KF, Weitz CJ. Physiological significance of a peripheral tissue circadian clock. Proceed National Academy Sci. 2008 Sep 30;105(39):15172-7.

8. la Fleur SE, Kalsbeek A, Wortel J, Fekkes ML, Buijs RM. A daily rhythm in glucose tolerance: a role for the suprachiasmatic nucleus. Diabetes. 2001 Jun 1;50(6):1237-43.

9. Ruiter M, La Fleur SE, van Heijningen C, van der Vliet J, Kalsbeek A, Buijs RM. The daily rhythm in plasma glucagon concentrations in the rat is modulated by the biological clock and by feeding behavior. Diabetes. 2003 Jul 1;52(7):1709-15.

10. Marcheva B, Ramsey KM, Buhr ED, Kobayashi Y, Su $\mathrm{H}$, Ko CH, et al. Disruption of the clock components CLOCK and BMAL1 leads to hypoinsulinaemia and diabetes. Nature. 2010 Jul;466(7306):627.

11. Pulimeno P, Mannic T, Sage D, Giovannoni L, Salmon P, Lemeille S, et al. Autonomous and selfsustained circadian oscillators displayed in human islet cells. Diabetol. 2013 Mar 1;56(3):497-507.

12. Rostom H, Shine B. Basic metabolism: proteins. Surg. 2018;36:4:153-8.

13. Morris Jr SM. Regulation of enzymes of the urea cycle and arginine metabolism. Ann Rev Nutrition. 2002 Jul;22(1):87-105.

14. Burgard P, Kölker S, Haege G, Lindner M, Hoffmann GF. Neonatal mortality and outcome at the end of the first year of life in early onset urea cycle disordersreview and meta-analysis of observational studies 
published over more than 35 years. J Inherited Metab Dis. 2016 Mar 1;39(2):219-29.

15. Morlion BJ, Stehle P, Wachtler P, Siedhoff HP, Köller M, König W, et al. Total parenteral nutrition with glutamine dipeptide after major abdominal surgery: a randomized, double-blind, controlled study. Ann Surg. 1998 Feb;227(2):302.

16. Bazinet RP, Layé S. Polyunsaturated fatty acids and their metabolites in brain function and disease. Nature Rev Neurosci. 2014 Dec;15(12):771.

17. Chen CH, Huang MH, Yang JC, Nien CK, Yang CC, Yeh $\mathrm{YH}$, et al. Prevalence and risk factors of nonalcoholic fatty liver disease in an adult population of taiwan: metabolic significance of nonalcoholic fatty liver disease in nonobese adults. J Clin Gastroenterol. 2006 Sep 1;40(8):745-52.

18. Shi J, Zhang Y, Gu W, Cui B, Xu M, Yan Q, et al. Serum liver fatty acid binding protein levels correlate positively with obesity and insulin resistance in Chinese young adults. PloS One. 2012 Nov 7;7(11):e48777.

19. Uysal KT, Scheja L, Wiesbrock SM, Bonner-Weir S, Hotamisligil GS. Improved glucose and lipid metabolism in genetically obese mice lacking aP2. Endocrinol. 2000 Sep 1;141(9):3388-96.

20. Mycielska ME, Patel A, Rizaner N, Mazurek MP, Keun H, Patel A, et al. Citrate transport and metabolism in mammalian cells: prostate epithelial cells and prostate cancer. Bioessays. 2009 Jan;31(1):10-20.

21. Donnelly KL, Smith CI, Schwarzenberg SJ, Jessurun J, Boldt MD, Parks EJ. Sources of fatty acids stored in liver and secreted via lipoproteins in patients with nonalcoholic fatty liver disease. J Clin Investigation. 2005 May 2;115(5):1343-51.

22. Tovar-Méndez A, Miernyk JA, Randall DD. Regulation of pyruvate dehydrogenase complex activity in plant cells. Eur J Biochem. 2003 Mar;270(6):1043-9.

23. Mashek DG. Hepatic Fatty Acid trafficking: Multiple forks in the road. Adv Nutr. 2013 Nov 6;4(6):697-710.

24. Erol E, Kumar LS, Cline GW, Shulman GI, Kelly DP, Binas B. Liver fatty acid binding protein is required for high rates of hepatic fatty acid oxidation but not for the action of PPAR $\alpha$ in fasting mice. FASEB J. 2004 Feb;18(2):347-9.

25. Kamagate A, Qu S, Perdomo G, Su D, Kim DH, Slusher S, et al. FoxO1 mediates insulin-dependent regulation of hepatic VLDL production in mice. J Clin Invest. 2008 Jun 2;118(6):2347-64.

26. Adamovich Y, Aviram R, Asher G. The emerging roles of lipids in circadian control. Biochimica et Biophysica Acta (BBA)-Molecular and Cell Biol Lipids. 2015 Aug 31;1851(8):1017-25.

27. Le Martelot G, Claudel T, Gatfield D, Schaad O, Kornmann B, Sasso GL, et al. REV-ERB $\alpha$ participates in circadian SREBP signaling and bile acid homeostasis. PLoS Biol. 2009 Sep 1;7(9):e1000181.

28. Lu TT, Makishima M, Repa JJ, Schoonjans K, Kerr TA, Auwerx J, et al. Molecular basis for feedback regulation of bile acid synthesis by nuclear receptors. Molecular Cell. 2000 Sep 1;6(3):507-15.

29. Han S, Zhang R, Jain R, Shi H, Zhang L, Zhou G, et al. Circadian control of bile acid synthesis by a KLF15-Fgf15 axis. Nature Commun. 2015 Jun 4;6:ncomms8231.

30. Simpson R. Drug therapy in patients with liver disease. In: Brown B, Ed. Anaesthesia for the patient with liver disease. Davis Company. 1981;57e76.

31. Yang X, Downes M, Ruth TY, Bookout AL, He W, Straume M, et al. Nuclear receptor expression links the circadian clock to metabolism. Cell. 2006 Aug 25;126(4):801-10.

32. Zhang YK, Yeager RL, Klaassen CD. Circadian expression profiles of drug processing genes and transcription factors in mouse liver. Drug Metab Dispos. 2008 Oct 6; 37:106-15.

33. Gachon F, Olela FF, Schaad O, Descombes P, Schibler U. The circadian PAR-domain basic leucine zipper transcription factors DBP, TEF, and HLF modulate basal and inducible xenobiotic detoxification. Cell Metabol. 2006 Jul 31;4(1):25-36.

Cite this article as: Alamri ZZ. The role of liver in metabolism: an updated review with physiological emphasis. Int J Basic Clin Pharmacol 2018;7:2271-6. 